\title{
The Recurrent Laryngeal Nerve and Thyroid Surgery; Who to Scope, When to Visualize, Who to Stimulate
}

Aruyaru Stanley Mwenda

Aga Khan University, Nairobi

Correspondence to: Dr. Aruyaru Stanley Mwenda, P.0 Box 30270-00100 Nairobi, Kenya.

Email:arumwesta@gmail.com

\section{Summary \\ Thyroid surgery is a common general and specialist surgical procedure. Recurrent laryngeal nerve injury during thyroid surgery, though rare, is the most feared surgical complication. Various steps and perioperative assessments can minimize recurrent laryngeal nerve injury. In this opinion article, two clinical vignettes are used at the introduction to discuss this topic. Literature is reviewed and conclusions made in the aspects of intra-operative recurrent laryngeal nerve exposure, the utility of intra-operative nerve monitoring and the use of peri-operative laryngoscopy}

where indicated. In conclusion, the literature shows that nerve exposure during thyroidectomy is a must. Nerve monitoring does not reduce the incidence of recurrent laryngeal nerve injury. All patients with pre-operative voice changes, cancer, prior head and neck surgery and those due for re-do thyroidectomy require pre-operative laryngocsopy.

Keywords: Recurrent Laryngeal Injury, Thyroidectomy, Nerve Exploration, Laryngoscopy

Ann Afr Surg. 2016;13(2): 72-6.

DOI: http://dx.doi.org/10.4314/aas.v13i2.9

\section{Introduction}

Thyroid surgery is a common general and specialist procedure (1). Larynx and trachea form the surgical bed irrespective of extent (1). Recurrent laryngeal nerve palsy is the feared complication post thyroid surgery (2). The recurrent laryngeal nerve is a branch of the vagus nerve carrying motor and sensory supply to the larynx. It has the sole motor supply to the sole abductor of the cord (posterior cricoarytenoid) (2). The following two clinical vignettes and the accompanying questions will assist us in the discussion of the topic of the recurrent laryngeal nerve during thyroid surgery. The aim of this article is to revisit the topics of nerve exploration during thyroid surgery, the need and indications for peri-operative laryngoscopy and role of intra-operative nerve stimulation.

\section{Case I}

A 49 year old female with no co-morbidities presented to our clinic after an incidental finding of anterior neck swelling during ENT review for short lived sore throat. She denied any symptoms of hyper- or hypothyroidism. She did not have pressure or infiltrative symptoms and her medical history was unremarkable. Examination was remarkable for a right nodular goitre. She was clinically and biochemically euthyroid. Ultrasound revealed multiple mixed nodules and image guided cytology showed suspicious follicular cells. She was scheduled for surgical lobectomy to get a histological diagnosis. Intra-operatively capsular dissection was carried out. The recurrent laryngeal nerve was visualized in the tracheoseophangeal groove and protected. Histology was benign. She complained of a sore throat the following day but was reassured and discharged. Six weeks later she presented again with persistent hoarseness and episodes of breathlessness. Indirect laryngoscopy showed immobile paramedian right vocal cord. 
What's the problem? Is her recurrent laryngeal nerve injured? Is this permanent or transient palsy? How should we proceed next?

\section{Case 2}

A 42 year old female with controlled asthma presented to our clinic with a long standing progressive anterior neck swelling. This was painless but was associated with recent onset of dysphagia and hoarseness. She denied any symptoms of hyper- or hypothyroidism. Examination revealed a right lobe nodular goitre with retrosternal extension. She was clinically and biochemically euthyroid. Neck ultrasound revealed an enlarged right thyroid lobe with mixed nodules and retrosternal extension. The fine needle aspiration cytology was benign. She was schedule for surgery based on her pressure symptoms. Pre-operative indirect laryngoscopy was normal. Intra-operatively a subcapsular right lobectomy was performed. The recurrent laryngeal nerve was not explored. She made uneventful post operative recovery. She is due for follow-up in the clinic.

What if she comes with hoarseness during the follow up visit? Should we do laryngoscopy during her next visit?

\section{Discussion}

\section{Anatomic Variations}

Anatomic variation is the main reason for most nerve injuries (1). These variations can occur in the pattern of division, relations of the nerve to the inferior thyroid artery and its branches and the existence of the nerve in a non-recurrent fashion (1-3). It is worth noting that up to 28 variations have been described in anatomy studies (2). Similarly, variations have been assessed relative to the ligament of berry and the tubercle of Zuckerkandl (3). But for the surgeon, it is easier to look at these variations relative to the inferior thyroid artery and its branches as the surgeon has to always address this artery as a step in thyroid surgery. One way is to classify the recurrent laryngeal nerve into type A (anterior to the artery), type B (between branches of the artery) or type $\mathrm{C}$ (posterior to the artery) $(1,2)$.

In a systematic review and meta analysis of 32 papers with 8655 recurrent laryngeal nerve sides assessing for the anatomic variation of the nerve relative to the inferior thyroid artery, Ling et al found the nerve to anterior (type A) in $20 \%$, in between the branches (type B) in $28 \%$ and posterior(type C) in $52 \%$ of the cases (1). These findings are reflected in a local dissection study by Kaisha et al which was included in the Meta analysis by Ling et al (3). In the same systematic review, the authors reviewed 21 articles with 38,568 recurrent laryngeal nerve sides searching for a nonrecurrent laryngeal nerve as a variation. They found the prevalence of a non-recurrent laryngeal nerve to be $0.57 \%$ (1). Although the sides were not indicated, it is common anatomic knowledge that a non-recurrent nerve is usually on the right owing to alterations in vascular embryology of the head and neck.

\section{Risk Factors for Recurrent Laryngeal Nerve Injury} Recurrent laryngeal nerve injury is classified as transient or permanent. Permanent injury is diagnosed after 12 months of persistent palsy (4). Permanent injury will occur in $0-6 \%$ of surgeries while transient injury/palsy occurs in $3-12 \%(1,4,5)$. The mechanisms of injury vary including ligation, traction, clamping, diathermy and ischemia (4). Transection occurs in only $0.3 \%$ of the injured nerves with majority of the injuries occurring on intact nerves (4). There's a slight preponderance to the right but this is not strongly supported as most of the documented injuries lack indication of the affected side (4). Various risk factors have been postulated to contribute to nerve injury (4). Firstly, re-operation increases risk of permanent injury by $2-30 \%$. This is due to altered tissue planes and difficult nerve visualization. In reoperation for cancer surgery, the rates of nerve injury are up to $6 \%$. Secondly, cancer surgery is associated with $20-50 \%$ increase in rates of nerve injury. This is contributed by tumour infiltration into or around the nerve (which occurs in up to $20 \%$ ) of cases. Thirdly, the extent of surgery contributes to increased risk of nerve injury. It is for example observed to be 12.6 times higher in total thyroidectomy compared with subtotal thyroidectomy $(4,6)$.

The surgeon experience is an argument that has been disputed as a contributor to nerve injury. Some accounts have argued that injuries are low under the hands of experienced surgeons (100 procedures per year or 45 nerves at risk per year) but this is contested by studies that show no difference in nerve palsy rates whether the surgeries are done by endocrine surgeons, well trained general surgeons in provincial hospitals or supervised trainees (4). In terms of laterality, we know that on the right side the nerve is more anteriorly positioned compared to the left (4). Most accounts of the injured nerves however don't document the laterality, and in the ones that do, there is no difference between left and right. Lastly, there is no evidence linking retrosternal goitre or Graves disease to increased risk of nerve injury, contrary to earlier arguments.

\section{Routine Intra-Operative Nerve Exploration}

The debate on whether or not to explore and visualize the nerve has now been put to bed. Surgeons argued in 
the past that exploration of the nerve would increase the risk of injury. That if you remained capsular you wouldn't stray into the course of the nerve $(7,8)$. Perhaps this was driven by the held believe that once injured, the recurrent laryngeal nerve could not be repaired (7). This changed in the late 1930s driven by the studies by Lahey of Boston and Ridell of London (7, 8). Between 1935 and 1938, Lahey started exploring the nerves and exposing them during thyroid surgery. At this time, the prevailing international rates of nerve injury stood at 3\% while in Lahey's clinic the nerve injury rates were $1.5 \%$ (7). He operated on 3000 nerves at risk and followed up the patients documenting any nerve injuries. As a result of this exploration, the nerve injury rate come down to $0.6 \%$. In conclusion he says; "As the result of exposing at least 3,000 recurrent laryngeal nerves in a period sufficiently long (three years) to permit late complications to occur if they were to occur, it may be said that the routine exposure of recurrent laryngeal nerves in thyroid surgery is a safe and justifiable procedure and will diminish, if not largely eliminate, injuries to that nerve" (7).

At the same time Ridell in London was not only exposing the nerves, he was doing laryngoscopy preand post-operatively (8). In his series of 1022 at risk nerves, Ridell does not compute any statistics, unlike Lahey, but he describes surgical steps that aid in nerve identification without injury (8). He identifies haemorrhage from the inferior thyroid artery as the single most agent contributing to nerve injury as the surgeon hurriedly ligates the artery to arrest bleeding (8). In 1970, in a narrative paper assessing his practice for 23 years, Ridell advocated not only for nerve identification but also for use of the nerve stimulator (a new device at the time) to limit injuries to the recurrent laryngeal nerve especially in bilateral surgery (9). He concludes by projecting a future that would be safe with the use of both nerve identification and stimulation during bilateral thyroid surgery: "If we weigh the hazards of injury to the recurrent laryngeal nerve on the scales of conscience and competence on the one side against non-identification and non-stimulation of the nerves on the other, the balance of probabilities is that the day will come when identification and stimulation become standard procedures in the bilateral operation" (8). These two propelled the surgical fraternity into routine exploration of the nerves.

In 2002, Hermann et al studied the incidence of nerve injury during surgery for benign disease over a 20 year period at a single centre in Austria (6). This study had three parts. Part 1 (1979-1990) included 9385 patients with 15865 nerves at risk. These were operated on without exposure of the nerves. In the second period (1990-1998) 6128 patients with 10548 nerves at risk were operated, this time it was routine to expose the nerves. The data in the two periods were compared and the findings were communicated to the surgeons in 1999. The third period (1999) included 930 patients with1561 nerves at risk that were operated on with routine exposure of the nerves, after the surgeons had had feedback from the findings of the prior 20 years.

The nerve injury rate reduced by the third period and was correlated to the extent of surgery (6). Locally in the country, the school of thought of not exposing the nerve still exists (unpublished). But a look at various standard general surgical textbooks will confirm that nerve exposure is one of the steps in thyroidectomy, not a point of debate (10-12).

\section{Intra-0p Nerve Monitoring}

Born in the 1970s to add to the push to reduce nerve injury, intra-op nerve stimulation is premised on the fact that not all intact nerves are functionally preserved, and that a surgeon's eye will only identity $1 / 10$ of the injured nerves (13). Higgins et al did a systematic review and meta analysis on the use of intra-op neuromonitoring compared with nerve visualization alone and their effect on post-operative vocal cord palsy. They carried out a literature search between 1966 and 2008. They included 1 randomized controlled trial, 7 prospective trials and 34 case series. In total they had 64699 nerves at risk. They found vocal cord palsy rates $3.52 \%$ in the neuromonitoring group versus $3.12 \%$ with visualization alone. The difference was not statistically significant (11). When a subgroup analysis assessing overall, transient and persistent palsy was done, not statistically significant difference was found (14). In another systematic review and meta analysis, Pisanu and colleagues looked at 3 randomized controlled trials, 7 prospective trials and 10 retrospective studies by searching the English literature up to 2013. These studies included 23512 patients with 35513 nerves at risk. All patients had pre-op and post-op laryngoscopy. For their meta analysis, 24038 (67.7\%) nerves at risk had intra-op neuromonitoring while 11475 had visualization alone. They assessed the outcomes in terms of overall, transient and permanent palsy. There were no statistically significant differences between the groups. For the subgroup analysis comparing transient and persistent cord palsy among high risk patients, no difference was found (5). The findings of these systematic reviews and meta analyses conclude that the use of intra-operative nerve monitoring does not reduce the incidence of recurrent laryngeal nerve 
injury when compared to visualization alone. It's up to the surgeon to decide if to use nerve stimulation as an adjunct (13). As such some associations recommend use of nerve stimulation only in high risk surgery and for learning purposes (15).

\section{Perioperative Voice Changes}

As in the second case vignette, some patients will present with hoarseness pre-operatively. A majority will have voice changes post operatively. It is estimated that $33 \%$ of patients will present with pre-op cord hypomotility in all diseases (4). Defective vocal cord apposition occurs in $70 \%$ of invasive cancers and $0.3 \%$ benign goitre (4). For benign diseases, $89 \%$ of cord dysfunction will recover post-operatively (4). Some causes of pre-operative hoarsenessincludehyperaemia (gastro oesophageal reflux), Reinkes oedema, chronic laryngitis and endotracheal intubation (vocal cord injuries occur in $30 \%$ of intubations) $(4,16)$.

Post operative hoarseness occurs in $30-87 \%$ of surgeries while post-operative dysphagia will occur in $50 \%$ of cases $(4,16)$. Causes include recurrent laryngeal nerve injury, external superior laryngeal nerve injury, cricoid muscle dysfunction, soft tissue swelling and intubation injuries $(4 \%$ of recurrent laryngeal nerve injury is due to intubation) (16). Vocal cord palsy may be present without voice change and vice versa. Sensitivity of voice changes in predicting cord paralysis greatly varies $(33-68 \%)(4,16)$. Preop cord assessment is necessary as it directs intraoperative decision making (16). Clinical evaluation of voice changes as a lone functional assessment of the recurrent laryngeal nerve is unacceptable as it will miss many unilateral cases (4).

\section{Perioperative Laryngoscopy}

As we have already discussed above, we cannot rely on voice assessment alone to infer recurrent laryngeal nerve function as this may be missed in unilateral cases (4). Laryngoscopy is the recommended examination for assessment of vocal cord position and function (4). Although fibre optic nasal endoscopy is the gold standard, indirect laryngoscopy is acceptable due to its widespread availability (4). Laryngoscopy performed by the anaesthetist at intubation is not adequate as it does not show movement but only position of the cord (4). So, who gets pre-operative laryngoscopy? Different risk stratifications have been developed to identify those patients who are at high risk of preoperative nerve dysfunction or intra-operative nerve injury. Table 1 highlights these risk strata and gives the recommendations for pre-op laryngoscopy from various bodies and authorities. All patients who undergo pre-op laryngoscopy should have post -op day 1 laryngoscopy if they have hoarseness. Any postoperative palsy requires serial exams until 12 months in order to establish if it is permanent or transient $(4,16)$.

$\begin{aligned} & \text { Table 1: Recommendations from Various Bodies on Use of } \\
& \text { Laryngoscopy in Thyroid Surgery (16) }\end{aligned}$
\begin{tabular}{|l|l|l|l|l|}
\hline $\begin{array}{l}\text { AMERICAN } \\
\text { HEAD/NECK } \\
\text { SOCIETY }\end{array}$ & $\begin{array}{l}\text { AMERICAN } \\
\text { THYROID } \\
\text { ASSOCIATION }\end{array}$ & $\begin{array}{l}\text { GERMAN } \\
\text { THYROID } \\
\text { ASSOCIATION }\end{array}$ & $\begin{array}{l}\text { BRITISH } \\
\text { THYROID } \\
\text { ASSOCIATION }\end{array}$ & $\begin{array}{l}\text { NATIONAL } \\
\text { COMPREHENSIVE } \\
\text { CANCER CARE } \\
\text { NETWORK }\end{array}$ \\
\hline All cancers & $\begin{array}{l}\text { Cancer with extra } \\
\text { thyroidal spread }\end{array}$ & $\begin{array}{l}\text { All patients } \\
\text { pre- and } \\
\text { post-op }\end{array}$ & All cancers & All cancers \\
\hline $\begin{array}{l}\text { Pre-op voice } \\
\text { changes }\end{array}$ & Pre-op voice changes & & $\begin{array}{l}\text { Pre-op voice } \\
\text { changes }\end{array}$ & \\
\hline $\begin{array}{l}\text { Re-do surgery } \\
\text { Re-do surgery }\end{array}$ & $\begin{array}{l}\text { Extensive cervical } \\
\text { adenopathy }\end{array}$ & & & \\
\hline $\begin{array}{l}\text { Prior neck/chest } \\
\text { surgery }\end{array}$
\end{tabular}

\section{Conclusion}

Recurrent laryngeal nerve injury during thyroid surgery is often due to variant anatomy, and mostly occurs during ligation of the inferior thyroid artery. Nerve exposure is one of the critical surgical steps in thyroidectomy. Routine use of intra-op nerve stimulation is not backed by evidence. All high risk patients require pre-operative laryngoscopy followed by post-op laryngoscopy if they develop hoarseness.

\section{References}

1. Ling XY, Smoll NR. A Systematic Review of Variations of the Recurrent Laryngeal Nerve. Clinical Anatomy. 2016; 29: 104-10.

2. Yalxcin B. Anatomic Configurations of the Recurrent Laryngeal Nerve and Inferior Thyroid Artery. Surgery. 2006; 139:181-7.

3. Kaisha W, Wobenjo A, Saidi H. Topography of the Recurrent Laryngeal Nerve in Relation to the Thyroid Artery, Zuckerkandl Tubercle and Berry Ligament in Kenyans. Clin Anat. 2011; 24: 853-7.

4. Hayward NJ, Grodski S, Yeung M, et al. Recurrent Laryngeal Nerve Injury in Thyroid Surgery: A Review. ANZ J Surg. 2013; 83:15-21.

5. Pisanu A, Porceddu G, Podda M, et al. Systematic Review with Meta Analysis of Studies Comparing Intra-Operative Neuromonitoring of Recurrent Laryngeal Nerves Versus Visualization Alone During Thyroid Surgery. J Surg Res. 2014; 181(1): 152-61.

6. Hermann M, Alk G, Roka R, et al. Laryngeal Recurrent Nerve Injury in Surgery for Benign Thyroid Diseases: Effect of Nerve Dissection 
and Impact of Individual Surgeon in More Than 27000 Nerves at Risk. Ann Surg. 2002; 235(2): 261-8.

7. Lahey FH, Hoover WB. Injuries to the Recurrent Laryngeal Nerve in Thyroid Operations: Their Management and Avoidance. Ann Surg. 1938; 108(4): 545-62.

8. Riddell VH. Nerves during thyroidectomy: A Comparison Between the Results of Identification and Non-identification in 1022 nerves exposed to Risk. Lancet. 1956; 268:638-41

9. Ridell V. Thyroidectomy: Prevention of Bilateral Recurrent Nerve Palsy. Br J Surg. 1970; 57(1): 1-11

10. Evers RM. Thyroidectomy (Lobectomy, Subtotal Resection, Total Thyroidectomy). In: Townsend CM Jr, Evers RM (Ed): Atlas of General Surgical Techniques. Philadelphia: Saunders, 2010 P 1-18

11. Wells SA Jr. Total Thyroidectomy, Lymph Node Dissection for Cancer. In: Fischer JE, Bland KI (Ed): Mastery of Surgery. Lippincott Williams \&
Wilikins 2007

12. Khatri VP, Asensio JA. Operative Surgery Manual. Philadelphia: Suanders 2007

13. Moris D, Vernadakis S, Felekouras E. The Role of Intraoperative Nerve Monitoring (IONM) in Thyroidectomy: Where Do We Stand Today? Surgical innovation 2014; 21(1):98-105

14. Higgins TS, Gupta R, Ketcham AS, Sataloff RT et al. Recurrent Laryngeal Nerve Monitoring Versus Identification Alone on Post-Thyroidectomy True Vocal Fold Palsy: A Meta-Analysis. Laryngoscope 2011; 121:1009-1017.

15. Royal Australasian College of Surgeons. Consensus Statement on Intra-Operative Electrophysiological Recurrent Laryngeal Nerve Monitoring During Thyroid Surgery. ANZ J Surg. 2014; 84: 603-5.

16. Sinclair CF, Bumpous JM, Haugen BR, et al. Laryngeal Examination in Thyroid and Parathyroid Surgery: An American Head and Neck Society Consensus Statement. Head \& Neck 2016; 38(6):811-9 